\title{
Characterization of Casting Defects in the Single Crystal Turbine Blade Airfoil
}

\author{
R. Albrecht ${ }^{a, *}$, W. Bogdanowicz ${ }^{a}$, J. Sieniawski ${ }^{b}$ And K. Kubiak ${ }^{b}$ \\ ${ }^{a}$ University of Silesia, Institute of Materials Science, Pułku Piechoty 1A, 41-500 Chorzów, Poland \\ ${ }^{b}$ Rzeszów University of Technology, Department of Materials Science, W. Pola 2, 35-959 Rzeszów, Poland \\ In the paper turbine blades made from single crystal CMSX-4 superalloy were investigated by X-ray diffraction \\ methods (topography, $\Omega$-scan mapping) complemented by scanning electron microscopy observations. By the X-ray \\ diffraction topography method several misorientation defects were visualized as well as dendrites arrangement. \\ It was discussed that tip of the airfoil and thin walled area of the turbine blades located near the trailing edge can \\ accumulate more growth defects than other airfoil part, due to the complex shape of the mould.
}

DOI: 10.12693/APhysPolA.130.1091

PACS/topics: 61.72.Ff, 61.72.--y, 61.66.Dk

\section{Introduction}

Increasing cost of rhenium and other refractory metals in CMSX-4 alloy are forcing aerospace industry to look for ways of reducing cost of turbine blades. Internal cooling or thinner airfoil sections reduces weight and cost of turbine blades and leads to development of thin walled blade structure [1]. However, if casting has thin walls it is formed by several dendrites so their mutual misorientation can lead to decrease in mechanical strength and should be well defined [2].

Nowadays, due to improvement in the directional solidification of turbine blades techniques the grain boundaries are almost entirely eliminated in the casts [3]. However, mechanism of dendrites growth and fluctuations of their growth directions are still poorly understood because of several number of factors affecting solidification process. Problematic predictions of casting defects in thin elements enables the development of new methods of dendritic structure analysis.

In this study, defects of crystal orientation complemented by microstructure observations of thin turbine blade airfoil parts were analyzed.

\section{Experimental}

Single crystal turbine blades were made from second generation industrial CMSX-4 superalloy. The casts were obtained with [001] axial orientation and at withdrawal rate of $3 \mathrm{~mm} / \mathrm{min}$ at the Research and Development Laboratory for Aerospace Materials in Rzeszów University of Technology. Two longitudinal cross-sections were prepared from airfoil region: sample SM-1 was cut near the leading edge of the blade airfoil and sample SM-2 was cut from trailing edge. Due to the geometry of the turbine blade airfoil samples have different inclinations to

\footnotetext{
*corresponding author; e-mail: ralbrecht@us.edu.pl
}

the blade axis $Z$. Therefore, plane of the sample SM-1 deviates by 2 degree from $Z$ axis to pressure side of the blade and plane of the sample SM-2 deviates by 3 degree to suction side.

The X-ray topography camera was set for backreflection and equipped with $\mathrm{Cu}$ micro-focus X-ray source [4]. To measure crystal misorientations samples were rotating during measurements \pm 2 degree (across 200 reflex). That geometry allows visualization of mutual misorientation of neighboring areas of relatively large single crystals samples with high degree of mosaic structure and has an accuracy of even arc minutes for detection of subgrain misorientation [5].

$\mathrm{X}$-ray topography is sensitive for several material factors such as local misorientation or $\gamma^{\prime}$ lattice parameter which depend on local chemical composition. Therefore, for proper interpretation of the topograms we applied additionally EFG $\Omega$-scan mapping [6] on prepared samples surface.

The defects of crystal orientation and lattice parameters were compared with the images of microstructure. Samples were not etched for directly comparison. All images of microstructure were obtained on scanning electron microscope (SEM) JEOL JMS 6480 in the backscattered electrons technique (BSE).

\section{Results}

The results of X-ray topography of samples SM-1 and SM-2 were presented in Figs. 1 and 2, respectively. Topograms were obtained with sample oscillation (Fig. 1a, 2a) and combined sample and film oscillation (Fig. 1b, 2b). The topogram from surface of the sample SM-1 (Fig. 1a) show fine straight contrast bands corresponding with primary dendrite arms (dendrite cores) growth direction. However, there are some regions with local misorientation defects marked as $\mathrm{C}$ and $\mathrm{L}$. The upper tip region $\mathrm{O}$ shows blurred contrast probably due to deformation of lattice. After applying film oscillation two low angle boundaries $G$ were visualized which crossed in 
the blurred area. It can be seen that the low angle boundaries begins to form during crystallization in direction $Z$ between dendrites marked as A and are visible only with film oscillation. The strain area $\mathrm{O}$ becomes larger on topogram with film oscillation (Fig. 1b). The X-ray topography results from sample SM-2 (Fig. 2a) shows short bands of contrast randomly arranged. Additionally, blurred contrast in the upper part $\left(\mathrm{M}^{\prime}\right)$ of the sample may be observed. After applying oscillation of film the two low angle boundaries appeared, marked as K. The topogram of sample SM-2 shows lower quality in compare to sample SM-1 probably due to the higher defects concentration. After applying film oscillation blurred area (P, Fig. 2b) appeared on the bottom of the sample.


Fig. 1. X-ray topograms from samples SM-1 without (a) and with film oscillation (b). Reflex $200 \mathrm{Cu} K_{\alpha}$ radiation. $Z$ - direction of crystallization.



Fig. 2. X-ray topograms from samples SM-2 without (a) and with film oscillation (b). Reflex $200 \mathrm{Cu} K_{\alpha}$ radiation. $Z$ - direction of crystallization.

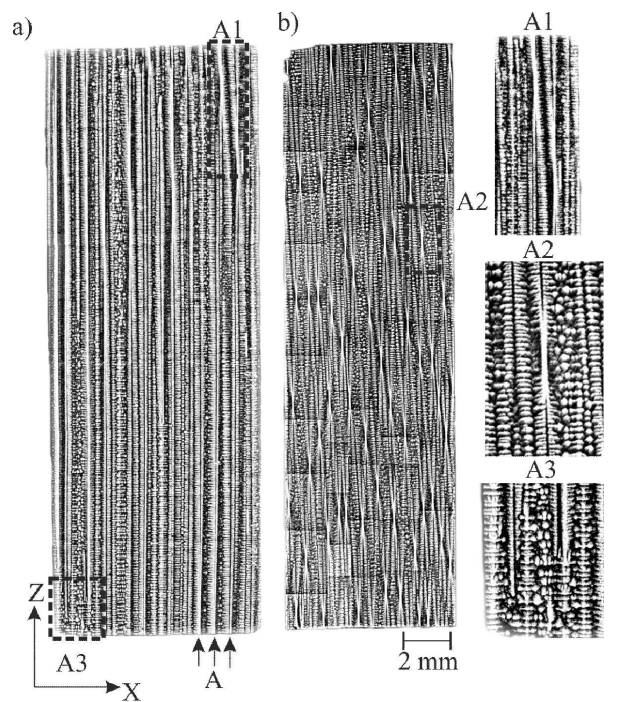

Fig. 3. SEM images of microstructure of sample SM1 (a) and SM-2 (b).

Figure 3 shows macro images of the sample surfaces obtained on SEM. On the surface of sample SM-1 the straight dendrite cores alignment can be observed. In the upper region of the sample the dendrites bending have occurred (inset A1, Fig. 3). On the other hand, sample SM-2 shows short parts of dendrite cores creating hourglass shape (inset A2, Fig. 3). The hourglass shapes are created when plane of the surface was cut with inclination to the dendrite cores growth. Moreover, some dendrites misalignment is also present in the area A3 marked on the topogram (inset A3, Fig. 3).

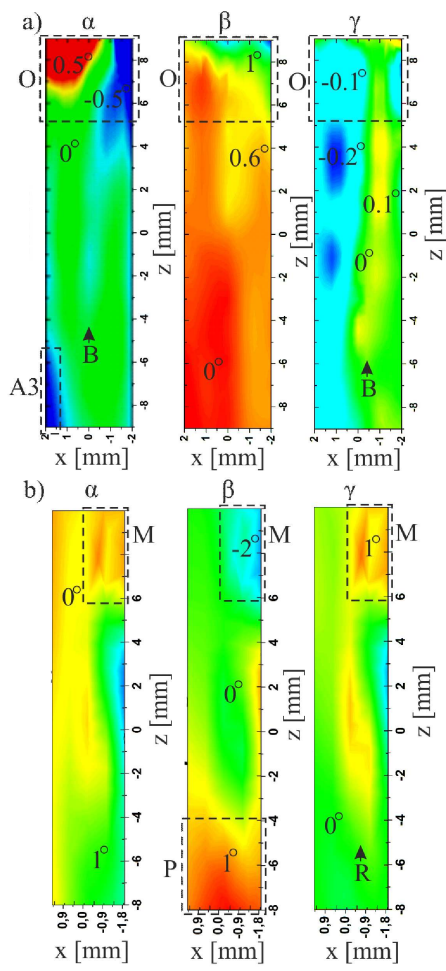

Fig. 4. EFG $\Omega$-scan maps of crystal misorientation of sample SM-1 (a) and SM-2 (b) surface. 
Figure 4 shows the results of orientation mapping conducted on the EFG diffractometer. Maps of crystal orientation of sample SM-1 (Fig. 4a) show few straight bands (B, Fig. 4a) along the surface which are corresponding to the topography results. The strain region $\mathrm{O}$ from topograms (Fig. 1) is divided into two parts with mutual misorientation of about 1 degree. Misorientation defects were also confirmed in the $\mathrm{C}$ area (Fig. 1a) on the rotation angle $\alpha$ (A3, Fig. 4a). Map of $\gamma$ rotation angle (Fig. 4a) visualizes two local defects placed similar to defects $\mathrm{L}$ on the topogram (Fig. 1a) with $0.2^{\circ}$ misorientation. Also changes in crystal orientation are visible in the low angle boundaries region (K, Fig. 1a) with maximum $0.5^{\circ}$ misorientation. Maps from sample SM-2 (Fig. 4b) shows higher degree of misorientation. Low angle boundaries are visible on $\gamma$ angle map (R, Fig. $4 \mathrm{~b})$ with about $1^{\circ}$ of misorientation. Strain region visible on the topograms (Fig. 2b) (P, Fig. 4b) appears on $\beta$ angle map of misorientation. Similar to topography results no straight lines representing dendrites cores were observed.

\section{Conclusions}

Conducted research proved that the tip of the turbine blade airfoil can accumulate more growth defects, probably caused by the shape of the mold. It was shown that mutual misorientation of neighboring sample areas occurs in all Euler angles. The thinner sample SM-2 cut from trailing edge of the blade gives more distorted topograms and higher degree of mutual misorientation. This is probably caused by differences in crystallization process in areas of different thickness and with different inclination of the mould wall. X-ray diffraction of thin part of the blade takes place more often with participation of secondary dendrite arms which are characterized by higher misorientation than primary dendrite arms. Additionally, changes between topograms of SM-1 and SM-2 samples may be associated with different orientation of diffraction vector relative to their surfaces. The Xray topography and diffraction mapping on EFG diffractometer proves to be the sufficient methods which used together, significantly improve precision in macroscopic scale crystal misorientation investigation in single crystal superalloys with dendritic microstructure.

\section{Acknowledgments}

This work was supported by the Polish National Centre for Research and Development (NCBiR) under Grant No. INNOTECH-K2/IN2/57/182858/NCBR/13.

\section{References}

[1] INSG Insight briefing paper: "Nickel-Based Super Alloys", Vol. 20, 2013.

[2] J.R. Hüttner, J. Gabel, U. Glatzel, R. Völkl, Mater. Sci. Eng. A 510-511, 307 (2009).

[3] R.C. Reed, The Superalloys: Fundamentals and Applications, Cambridge University Press, 2008.

[4] J. Auleytner, Acta Phys. Pol. A 17, 111 (1958).

[5] W. Bogdanowicz, Scr. Mater. 37, 829 (1997).

[6] H. Berger, H.-A. Bradaczek, H. Bradaczek, J. Mater. Sci. Mater. Electron. 19, 351 (2008). 